\title{
Open surgical repair of a giant abdominal aortic aneurysm
}

\author{
Görkem Yiğit, Ferit Çetinkaya, Bahadır Aytekin, Mehmet Ali Türkçü, Anıl Özen, Ahmet Sarıtaş \\ Department of Cardiovascular Surgery, Ankara City Hospital, Ankara, Turkey
}

Kardiochir Torakochir Pol 2019; 16 (3): 144-146

Abdominal aortic aneurysm (AAA) is more common at 50-60 years of age although it can be seen in any age stage of life [1]. The most important factor in the pathogenesis is elastin and collagen degeneration in the media layer. Aneurysm may be associated by collagen degeneration or autoimmune diseases such as Marfan syndrome, Takayasu's arthritis, giant cell arthritis, temporal arthritis and Behçet's disease [2]. The majority of cases are asymptomatic and are detected during physical examination or imaging performed for other reasons.

AAA presents as back pain, abdominal pain, leg pain or abdominal distention. Ultrasonography (USG), computed tomography (CT) or magnetic resonance imaging (MRI) is used for diagnosis. The most serious complication of AAA is rupture. Mortality rates in aortic rupture reach up to $90 \%$ [3]. The risk of rupture is high in aneurysms greater than $5 \mathrm{~cm}$, aneurysms growing faster than $0.5 \mathrm{~cm}$ per year or in painful AAAs. Treatment is indicated for patients in this group either with open surgery or an endovascular method [3].

A case of infrarenal AAA, with a diameter of $15.8 \mathrm{~cm}$ in a 64-year-old male patient, is presented here. Open surgical repair using an aorto-biiliac $Y$ graft interposition was opted for and the patient was discharged successfully on the tenth postoperative day.

A 65-year-old male patient with complaints of abdominal bloating, severe abdominal and back pain and a palpable mass in the abdomen was referred to the emergency department. His past medical history included hypertension, chronic kidney disease, chronic constipation and coronary artery bypass grafting. He had ignored his symptoms and has not applied to any hospitals for 2 years. Abdominal USG revealed an infrarenal abdominal aortic aneurysm with a diameter of $9.5 \mathrm{~cm}$.

On physical examination, blood pressure was $125 / 80 \mathrm{~mm} \mathrm{Hg}$ and pulse was $80 /$ minutes. The bowel sounds were normal. There was a pulsatile mass in the umbilical region that was tender on palpation. Upper and lower extremity distal pulses were palpable. Neurological examination was normal.

The patient was followed up in the intensive care unit. Following arterial and rhythm monitoring, blood pressure and rhythm control were achieved by intravenous beta blocker administration. Opioid pain killers were preferred for the pain control. Monitoring of the hematocrit levels at 2-hour intervals did not display any decrease. The patient underwent thoracoabdominal CT angiography, which revealed an infrarenal AAA, with a diameter of $158 \mathrm{~mm}$, surrounded by an intraarterial mural thrombus (Figure 1). Since the aneurysm presented 90 degrees between the pouch neck and the pouch, due to both the width of the pouch and the size it reached, it was decided to perform open surgical repair. Coronary angiogram via the right radial artery showed no stenosis other than $70 \%$ lesion in the right coronary artery (RCA). Since preoperative antiaggregant treatment was not appropriate, percutaneous coronary intervention was not performed for the lesion at the RCA.

The patient underwent surgery on the $2^{\text {nd }}$ day of intensive care follow-up, under elective conditions. A midline laparotomy was performed and the abdominal aorta was exposed. The aorta was encircled by surgical tape in the infrarenal region. Bilateral external and internal iliac arteries were also encircled by surgical tapes. Following the placement of clamps, aneurysmal sac was opened and $25 \times 15 \mathrm{~cm}$ diameter mural thrombus was removed (Figure 2). Interposition of a Dacron graft between the abdominal aorta and bilateral external iliac arteries was performed (using a bifurcated $20 \times 10 \mathrm{~mm}$ aortoiliac graft). The right internal iliac artery was anastomosed from the proximal part of the bifurcated graft using a separate graft. The left internal iliac artery that reached $8 \mathrm{~cm}$ in diameter was ligated using a strip.

The examination and USG findings 1 month after surgery were normal. Peripheral arterial flow was detected as triphasic and the patient was free of claudication. The patient complained of erectile dysfunction and ejaculation problems. Hence, the patient was referred to the urology clinic.

Infrarenal AAA is seen in $2-5 \%$ of males over 60 years of age [4]. AAAs are asymptomatic in most patients. Abdominal examination and abdominal USG are of vital importance in the diagnosis of AAA.

Currently, the majority of patients with AAA (60-70\%) are treated with endovascular aneurysm repair (EVAR) [5].

Address for correspondence: Görkem Yiğit MD, Department of Cardiovascular Surgery, Ankara City Hospital, Ankara, Turkey,

e-mail: drgorkemyigit@gmail.com

Received: 19.05.2019, accepted: 28.08.2019. 

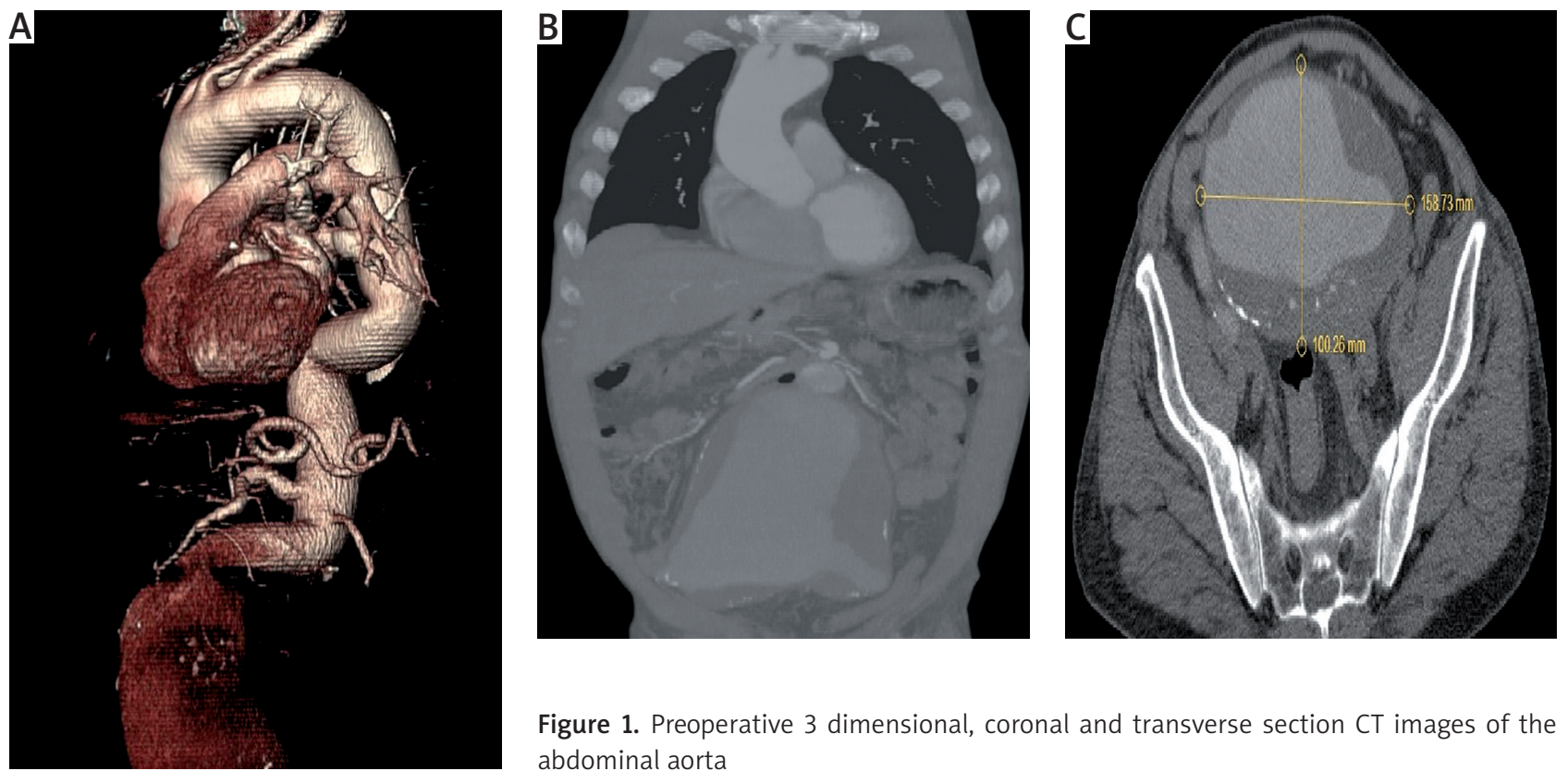

Figure 1. Preoperative 3 dimensional, coronal and transverse section CT images of the abdominal aorta
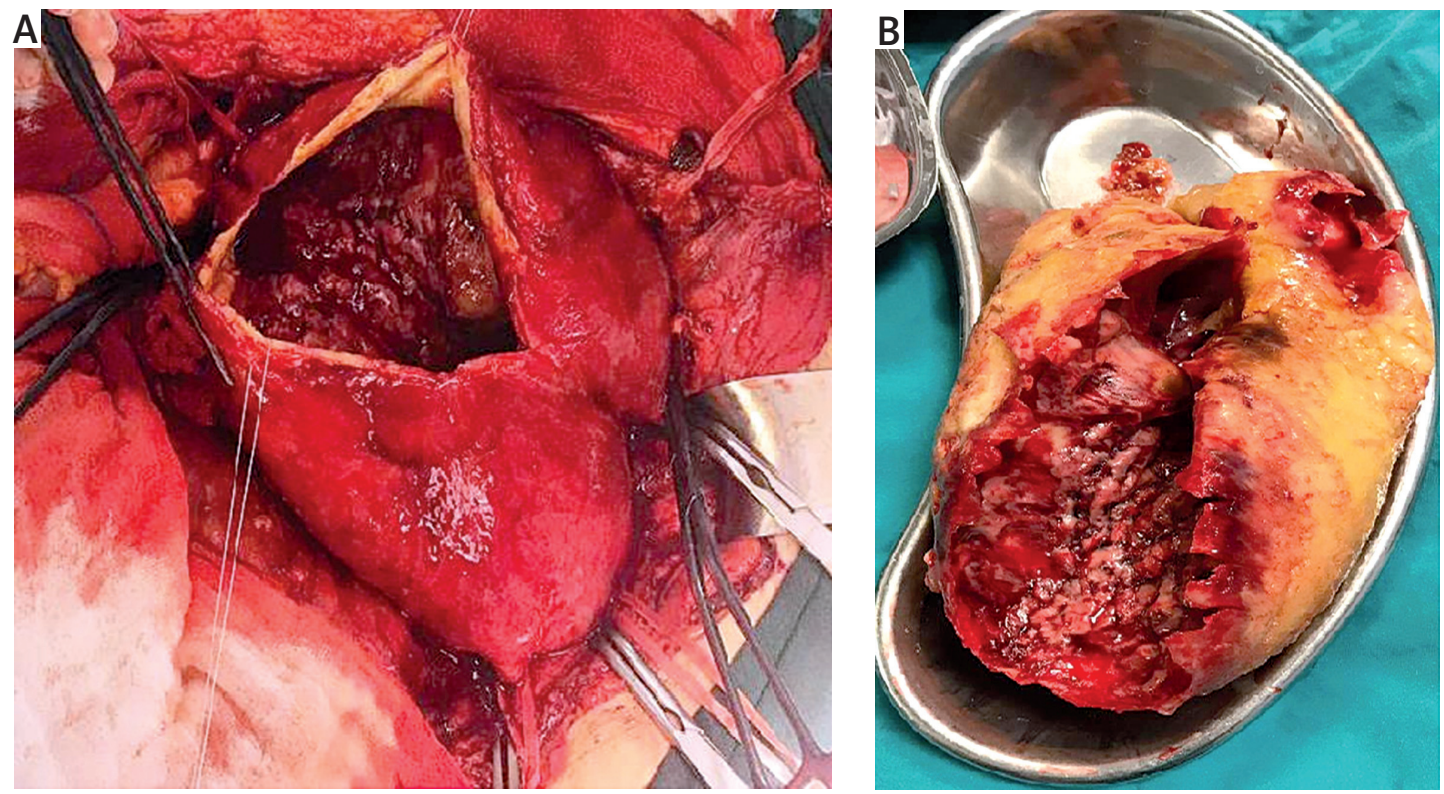

Figure 2. Aneurysmal sac containing a mural thrombus of approximately $25 \times 15 \mathrm{~cm}$

EVAR treatment cannot be applied to every patient, although it is less invasive. One of the reasons that EVAR was unsuitable for this patient was due to the giant size $(15.8 \mathrm{~cm})$ of the aneurysm. However, the iliac arteries being surrounded by mural thrombi is another factor that complicated endovascular intervention. If there are no anesthetic or medical contraindications for patients with nonruptured AAAs, open surgical repair should be the choice of the treatment according to the NICE guidelines [6] published in 2018.

Although the aneurysm diameter is the most important factor triggering abdominal aortic aneurysm rupture, low atmospheric pressure and the presence of low serum elastin-derived peptides have also been reported as addi- tional factors $[7,8]$. When Bown et al. reviewed 580 cases of rupture over a 10-year period, they found a significant elevation in ruptured AAA admissions in December (when atmospheric pressure was the lowest) compared to August (when atmospheric pressure was the highest) [7]. This case was also referred to our hospital in December and the severe abdominal and back pain on admission mimicked a ruptured AAA.

Open surgical repair is associated with higher incidence of complications when compared to EVAR. Erectile dysfunction occurring in our patient following surgery may be due to the ligation of the internal iliac artery. Ligation of the iliac artery is performed in gynecological, oncological and vascular surgery for various reasons. Complications of in- 
ternal iliac artery ligation in vascular surgery patients include hip claudication (21.2\%), spinal cord ischemia (9\%), colonic ischemia (7\%), hip necrosis (5\%) and erectile dysfunction (2.7\%) [9]. The complication of erectile dysfunction in this case may have also been caused by injury to the sacral parasympathetic ganglia. There are more cases of complications due to internal iliac artery ligation following vascular surgery than obstetric surgery reported in the literature [9]. This may be due to the fact that vascular surgery patients are more prone to obstructive atherosclerosis with older and developing collaterals. As a matter of fact, in this case, the age of the patient is 65 and he also had coronary artery disease in which atherosclerosis seems to be the most important factor.

According to a study about giant abdominal aortic aneurysms (>13 $\mathrm{cm}$ ) by Ullery et al., it was observed that nearly all patients were male, like the patient we present here [10]. This may be due to the association of estrogen receptors concentrated in vascular cells, particularly in women with low metalloproteinase activity [11].

Although the incidence of giant AAA is very low nowadays, most of these patients are treated with classical open surgery because of anatomical obstacles. Our case is one of the largest case of AAAs in the literature $(15.8 \mathrm{~cm})$ and was successfully treated with open surgery.

\section{Disclosure}

The authors report no conflict of interest.

\section{References}

1. Amunden S, Trippestad VA, Soride O. Abdominal aortic aneurysms a national multicenter study. Eur J Vasc Surg 1987; 1: 239-243.

2. Svensson LG, Crawford ES. Aortic dissection and aortic aneurysm surgery: clinical observations, experimental investigations and statistical analyses. Part III. Curr Probl Surg 1993; 30: 1-163.

3. Cleveland Clinic. [https://my.clevelandclinic.org/departments/heart/about].

4. Darling RC, Messina CR, Brewster DC. Autopsy study of unoperated abdominal aortic aneurysm. Circulation 1977; 56 (Suppl 2). II-161.

5. Levin DC, Rao VM, Parker L, Frangos AJ, Sunshine JH. Endovascular repair vs open surgical repair of abdominal aortic aneurysms: comparative utilization trends from 2001 to 2006. J Am Coll Radiol 2009; 6: 506-509.

6. Abdominal aortic aneurysm: NICE guideline DRAFT (May 2018).

7. Bown MJ, McCarthy M, Bell F, Sayers RD. Low atmospheric pressure is associated with rupture of abdominal aortic aneurysms. Eur J Vasc Endovasc Surg 2003; 25: 68-71.

8. Petersen E, Gineitis A, Wagberg F, Angquist KA. Serum levels of elastin-derived peptides in patients with ruptured and asymptomatic abdominal aortic aneurysms. Eur J Vasc Endovasc Surg 2001; 22: 48-52.

9. Chitragari G, Schlosser FJ, Chaar C, Sumpio BE. Consequences of hypogastric artery ligation, embolization, or coverage. J Vasc Surg 2015; 62: 1340-1347.

10. Ullery BW, Itoga NK, Lee JT. Giant abdominal aortic aneurysms: a case series and review of the literature. Vasc Endovasc Surg 2015; 49: 242-246.

11. Laser A, Ghosh A, Roelofs K, Sadiq O, McEvoy B, DiMusto P, Eliason J, Upchurch GR Jr. Increased estrogen receptor alpha in experimental aortic aneurysms in females compared with males. J Surg Res 2014; 186: 467-474. 